\title{
Subacute sclerosing panencephalitis as a cause of chronic dementia and relapsing brain disorder
}

\author{
M. DONNER, O. WALTIMO, J. PORRAS, H. FORSIUS, AND \\ A.-L. SAUKKONEN \\ From the Department of Neurology, University of Helsinki, the Department of Paediatrics, \\ University of Oulu, and the Children's Clinic of the University Central Hospital \\ of Helsinki, Finland
}

SUMMARY Two patients with subacute sclerosing panencephalitis (SSPE) are described because of the course of the disease, which is still thought to be unusual but might prove to be rather common. $\vec{O}$ In both cases there was apparent recovery after about two years of illness. One of the patients had a relapse after eight years of remission and seems to have recovered also from this second period of $\vec{\omega}$ illness, though with a more severe mental defect than after the first one. Thus, SSPE should be kept $\frac{\rho}{5}$ in mind as a possible aetiological factor in patients with a persisting slight to severe dementia after a brain disorder. Apparent recovery from SSPE does not exclude the possibility of relapse even many $\mathfrak{c}_{\mathrm{N}}$ years later.

It was formerly thought that subacute sclerosing panencephalitis (SSPE) progressed steadily until the death of the patient. A more chronic type of the disease seems to be more usual nowadays, possibly due in part to better care of the chronically ill patient. The patient goes through the different stages of the disease but can remain in a 'burned out' stage for many years, fully disabled both mentally and physically. SSPE should be kept in mind as a cause of chronic neurological disease in children and young adults. The diagnosis can usually be made by serological and CSF examinations (determination of measles antibody and platelet agglutination tests, examinations of the amount and fractions of gammaglobulin) and reappraisal of EEGs registered during the first stages of the disease. Patients with the chronic type of the disease may have partial remissions lasting some weeks to months.

Zeman and Kolar (1968) state that remissions are common and may extend over several years. A few cases have been reported in detail of apparent recovery, at least for many years, though with a lasting mental defect. Cobb and MorganHughes (1968) described two young men with SSPE with typical progressive mental symptoms, confirmed by the presence of periodic complexes in the EEG, a paretic Lange curve in the CSF, and brain biopsy findings which were typical in one case and equivocal in the other.
The neurological symptoms were very slight and neither patient had any regular recurrent jerkg Both recovered to a large extent and have been $\frac{D}{0}$ followed after recovery for eight and two years, respectively. At the follow-up examination the $\overrightarrow{0}$ first patient was mentally slightly retarded, th other showed only slightly defective intelligence. In both the results of psychological performance tests were much poorer than those of verbal tests. The EEG no longer showed any periodic complexes and the background activity had $\stackrel{\AA}{\varrho}$ improved.

Resnick, Engel, and Sever (1968) describe a well-documented case of SSPE in a 15 year old boy. There was a typical clinical course with myoclonic jerks, periodic complexes in the EEG, pathological colloidal gold curve of the CSF, elevated measles antibodies in the CSF, and 3 . typical findings at brain biopsy. After one and a half years of progressive illness, recovery began $₹$ and had lasted for two and a half years without 0 relapses at follow-up examinations. The young man was slightly disabled by a spastic hemiparesis and hemianopia and also had a speech disturbance. Mentally he was rather severely retarded. The follow-up EEG showed no periodic complexes. The measles antibody titre $\mathrm{\omega}$ in the CSF was still elevated $(1: 32, \mathrm{CF})$.

Kennedy (1968) mentions briefly an 11 year old girl with SSPE. The repetitive spasms and $\stackrel{\mathscr{\perp}}{\rightarrow}$ mental symptoms were alleviated to such a 
degree that she was able to return to school. Details are not given.

Legg (1967) reported a case of 'good recovery' with a follow-up time of four years without relapses. The measles antibody titres were followed and the complement fixation test returned to normal (from $1: 40$ to $1:<10$ ) but the haemagglutination inhibition titre remained elevated (1:32 and 1:64 respectively). Other details are not given.

Landau and Luse (1958) describe a case of a 10 year old boy with SSPE for eight years. Before it caused his death he had two periods of partial recovery for two years and one year. During these remissions he learned to walk again and speak short sentences.

In the three cases of recovery from an acute disease thought to have been SSPE described by Kurtzke (1956), Simpson (1961), and Pearce and Barwick (1964) the diagnosis is uncertain. None of them fulfilled more than one of the four commonly used criteria for the diagnosis of SSPE (repetitive movements, repetitive complexes in the EEG, high levels of gammaglobulins in the cerebrospinal fluid, and high antibody titres against measles).

During the 10 year period 1960-70 in Finland 23 cases of SSPE have come to our knowledge and one or more of us has personally seen 19 of these patients (Pettay, Donner, Halonen, Palosuo, Salmi, and Tiilikainen, 1971). Five of them have had a subacute disease ending fatally. A chronic course extending over many years occurred in 15 cases. Three of these have had partial shortlasting remissions for some weeks to months and two show some mental recovery after five years of severe illness, though physically they are still totally disabled. One is a new case and two of the 23 cases belong to the third type with apparent recovery mentioned in the introduction.

\section{CASE 1}

The patient, a girl born 10 August 1950, is the fourth of four siblings. Her sibs are healthy, but the father is a heavy drinker and the mother was under hospital treatment in the summer of 1969 with a diagnosis of involutional melancholia. The patient weighed 3,050 $\mathrm{g}$ at birth. Development during early childhood had been normal; she had learned to walk at 13 months and to talk just before the age of 2 years. On entering school she already knew the alphabet and was able to spell a little. Her health had been good before going to school except for rather severe whooping cough at the age of 4 , but her family had no knowledge of measles in her history.

The first two years in school passed fully normally but in the third form, during the spring term of 1959 , she clearly had difficulties and the first nocturnal convulsion occurred towards the morning of 4 March 1959. After three similar fits in the course of the spring she was sent on 6 April 1959 to the Children's Clinic of the University Central Hospital of Helsinki for examination. She was subsequently admitted for control examinations a number of times, the latest in the summer of 1961. The patient's neurological status was found to be normal every time, except for suspected stasis in the eyegrounds and mention of mental slowness in the case reports. Routine blood and urine tests were normal, as also were radiographs of the skull and the field of vision. The cerebrospinal fluid analyses and EEG findings are given in Table 1 and Fig. 1. The diagnosis was 'convulsions' probably due to encephalitis.

TABLE 1

RESULTS OF CEREBROSPINAL FLUID ANALYSES IN TWO CASES OF SUBACUTE SCLEROSING PANENCEPHALITIS

\begin{tabular}{|c|c|c|c|c|c|}
\hline $\begin{array}{l}\text { Date of } \\
\text { examination }\end{array}$ & $\begin{array}{c}\text { Protein } \\
(\mathrm{mg} / 100 \mathrm{ml} .)\end{array}$ & Nonne & Pandy & $\begin{array}{c}\text { Leuc. } \\
\text { per } \\
\text { c.mm }\end{array}$ & $\begin{array}{c}\gamma-\text { Globu- } \\
\text { lin } \mathrm{mg} / \\
100 \mathrm{ml} . \\
(\%)\end{array}$ \\
\hline
\end{tabular}

\section{Case 1}

17 Apr. 1959

28 Apr. 1959

18 June 1959

16 Dec. 1959

12 Nov. 1960

24 Feb. 1961

15 June 1961

28 Mar. 1970

24 Apr. 1970

11 May 1970

27 May 1970

80
80
95
80
80
80
90
282
112
102
96

+
+
+
+
+
+
+
+

+
+
+
+
+
+
+

$\begin{array}{rr}5 & \\ 5 & \\ 4 & \\ 5 & \\ 1 & \\ 4 & \\ 1 & \\ 22 & 133(47) \\ 1 & 46(41) \\ 1 & 34(33) \\ 2 & \end{array}$

Case 2

7 June 1966

11 July 1966

28 Apr. 1970

34
32
14

$3,6(26)$

In addition to rare attacks of unconsciousness and convulsions occurring in the early morning, she began in December 1960 to have momentary falling fits, sometimes several in the course of a day, and outbursts of rage were a new feature. At this stage her progress in school was so poor that she was transferred to a school for mentally retarded children, the course at which she completed in due time. The attacks having thus changed in character, the patient was examined in the spring of 1961 at the Neurosurgical Clinic of the University Central Hospital of Helsinki. Pneumoencephalography and left carotid angiography gave normal findings.

She was under the observation of the Child Guidance Clinic of Central Finland from summer 
FIG. 1. Case 1, R.P. I. EEG in April 1959, one month after onset of convulsions and some months after beginning of mental deterioration. Background activity is irregular and slightly slow. Periodic slow wave complexes recur at intervals of 6 to 35 sec (sometimes the interval may be up to $\left.1_{2}^{1} \mathrm{~min}\right)$. Two months later the interval was regular, 3 to 6 sec. II. February 1961, during the worst period of the disease. The periodic complexes recur at intervals of 3 to $10 \mathrm{sec}$. There are now bursts of sharp waves associated with the slow waves. The background activity is irregular and of low amplitude, but there is still some alpha activity. III. In August 1963, during the period of recovery, the background activity is still irregular but there is much alpha activity. Some generalized bursts of irregular spikes and waves. Sometimes irregularly recurring sharp and slow wave complexes which have some resemblance to the periodic complexes in the earlier EEGs.-In 1964, 1965, and 1967 there is more regular alpha activity, and every now and then series of generalized sharp and slow wave complexes occur. IV. March 1970, one month after beginning of the relapse. The background activity is more irregular. Small generalized bursts of 4 to $9 \mathrm{~Hz}$ waves of higher amplitude are seen. These usually come quite irregularly but sometimes, as in the Figure, at intervals of 5 to $6 \mathrm{sec}$.

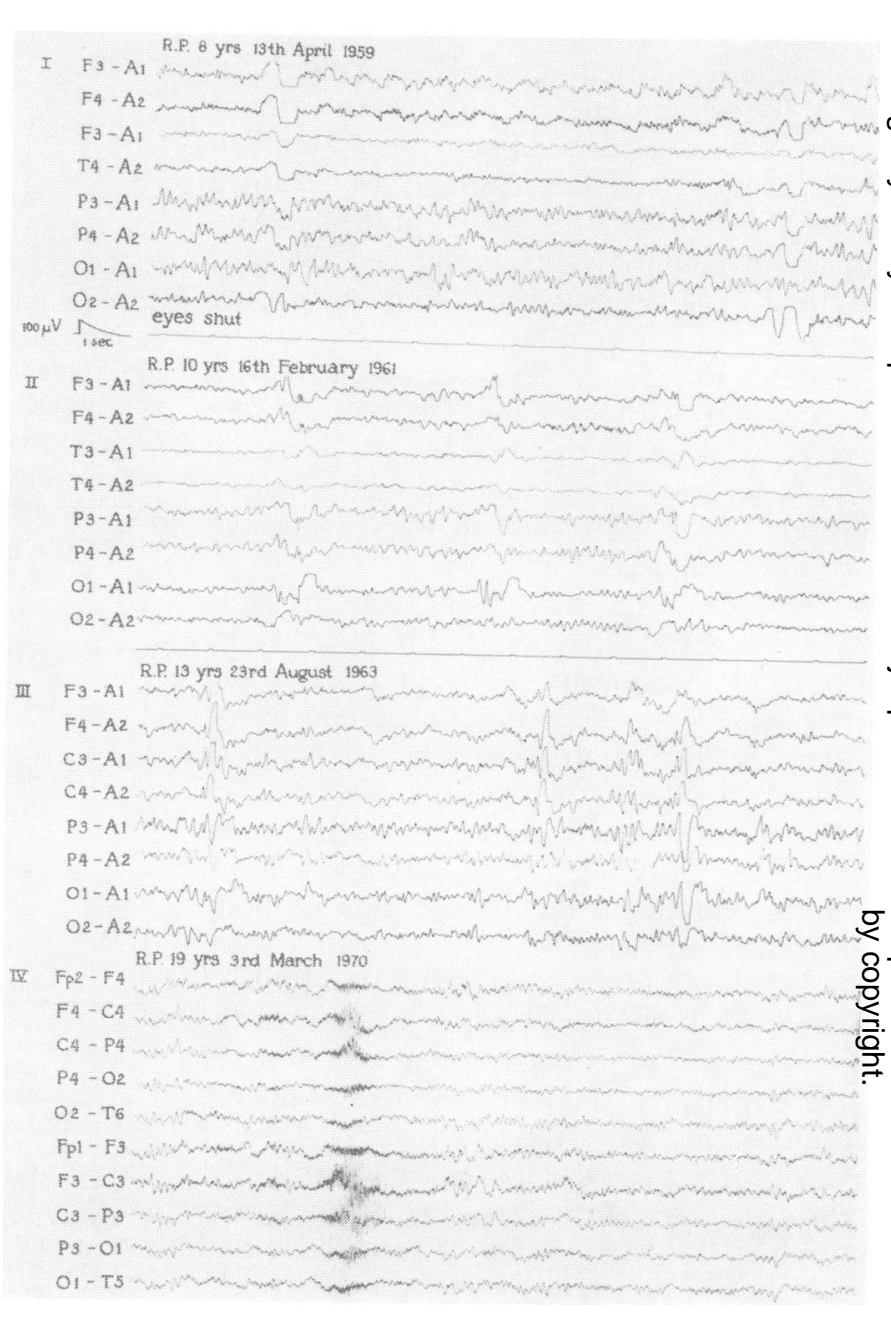

1961 to autumn 1966. The records mention at first fairly frequent minor fits, then a decrease in their frequency, and after the autumn of 1963 no minor attacks. On the other hand, some major nocturnal attacks still occurred occasionally except for the last two years. Since finishing school the patient has worked as a housemaid, though not for very long in any one place.

Since the spring of 1969 the patient has attended the Mental Health Office of Central Finland with a diagnosis of slight mental retardation. According to the records she had still seemed quite as usual on 20 January 1970, but in February 1970 her condition underwent conspicuously rapid deterioration, her speech became dysarthric, there were disturbances of vision, and headache, vomiting, and staggering. She was therefore admitted to the Central Hospital of
Central Finland on 19 March 1970 on a suspicion of encephalitis.

At this stage the patient was found to have diffi culties with regard to body image, the right uppero extremity had a tendency to downward pronation and Babinski's sign was doubtful on the left side.을 Gamma radiography with technetium was suspicious of a tumour in both occipital regions, but seleniumo scanning showed nothing abnormal. Vertebral angiography was interpreted as normal.

Because of the findings in the cerebrospinal fluid N and the EEGs (Table 1 and Fig. 1) the patient was స్ట transferred to the Department of Neurology, Uni-O versity of Helsinki. Clinical examination showed a young woman of large and robust build who did not? seem to be very well aware of where she was or of the month and year. She confused the right and left 
sides and was unable to read, write or calculate. The margins of the optic discs were slightly diffuse and there was possible homonymous hemianopsia on the right. The tendon reflexes seemed slightly brisk though Babinski's sign was absent on both sides and the abdominal reflexes were elicited. She also had very slight tremor of the hands. There were no repetitive myoclonic jerks.

Titres of measles antibody were $1: 1024$ and $1: 2048$ (CF) and 1:2560 (PA) in serum and 1:32 (CF) and $1: 320$ (PA) in cerebrospinal fluid. Left carotid angiography suggested slowed circulation in the post parietal region.

ACTH therapy was started. It did not appear to be of any help but during her stay in the University Central Hospital there was no progression of the disease. The patient was able to return home.

The first psychological examination was made in 1959, when the IQ was 68 (Wechsler). The verbal test component was 77 and the performance test component 64. Control examinations in 1960, 1961, and 1962 gave IQs of 83, 75, and 74 (TML), respectively. In May 1969 she had an IQ of 59 (Waiss), the verbal test component being 69 and the performance test component 50. In April 1970 severe spatial agnosia was evident and the verbal IQ was 50 (Wisc).

\section{CASE 2}

This patient, a boy born on 27 July 1959, is the fifth of seven siblings. Gestation, delivery, and early development had been normal. His health had been good in early childhood, though he had had febrile pharyngitis three times at the age of 2 to 4 years. When he was 2 years of age there had been measles in the family but there was no recollection of his having contracted it.

In May 1966 the patient began to have jerks that were repetitive at fairly regular intervals of about five seconds. At the same time he became disobedient, changed from sinistrality to dextrality, and stumbled with his left foot on walking. Within two months his ability to understand speech deteriorated and he became infantile.

During this initial stage the patient also had varicella and was admitted to the Department of Pediatrics, University of Oulu for examination. The neurological findings were staggering gait, rigidity of the left upper extremity, and uncertainty in coordination tests, in addition to the features already reported in the case history.

After this there was continuous slow progression of the disease, until rapid deterioration occurred in May 1967. He was now unable to walk, swayed even when seated, and had numerous involuntary movements of the extremities. Speech was thick and faltering, and dysphagia was observed. The worst stage of the disease was in September 1967, when he was drowsy, unable to turn in bed, and could not control his urine or faeces.

During the autumn of 1967 clear signs of remission were noted and in January 1968 he re-learned to walk. In May he rode a tricycle, and in the course of the spring he began again to form sentences. At this phase the repetitive jerks also stopped. In the summer of 1968 he no longer was incontinent and the mental absences of one to two minutes' duration formerly occurring in the morning disappeared.

In October 1968 he showed clumsiness of fine movements, the left hand being the poorer, and the co-ordination tests were not possible. At the control examination in April 1970 his general physical condition and external appearance corresponded to his calendar age, but definite perceptional disturbances were evident; however, coordination tests were successfully performed and fine movements were practically normal, as also were muscular tone and tendon reflexes.

The EEG and CSF findings are shown in Table 1 and Fig. 2, carotid angiography, pneumoencephalography, and isotope scanning of the brain were done in the summer of 1966 but nothing abnormal was found. The serum measles antibody titres were determined in September 1968 as 1:512 (CF), and in April 1970 1:1024 (CF) and 1:1280 (PA). The measles antibody titre in the cerebrospinal fluid was 1:16 (CF) in September 1968.

Psychological tests were carried out in July 1966, October 1968, and April 1970. At the first time the IQ was 79 (TML) and the verbal test component was notably better than the performance test component. In 1968, although the IQ was 43 (TMH-TML) and the profile very similar to the first one, he concentrated better on his tasks and was more interested. In the spring of 1970 he had an IQ of 58 (TML). He was speaking fluently and thinking was rather logical, but the results of the performance tests continued to be quite poor-for example, it was impossible for him to solve any of the WISC performance tasks and the circle was the only shape he was able to draw.

\section{DISCUSSION}

Neither of these two patients has been subjected to brain biopsy; in other respects they fulfil the criteria for a diagnosis of SSPE. Thus, they showed intellectual and personality changes, case 2 had repetitive myoclonic jerks and case 1 frequent falls possibly due to such jerks or to the atonic phase which is part of the typical movement (Pampiglione, 1964). Both had the characteristic EEG changes with periodic complexes, and in the cerebrospinal fluid the gammaglobulin was elevated. The measles antibody titres were high in the serum and in the CSF. 
FIG. 2. Case 2. S.M. I. EEG in December 1966, half a year after onset of the symptoms. Background activity is very irregular. Slow wave focus in the right hemisphere. Every 3 to 4 sec there occur generalized slow waves with some sharp waves, stronger on the right side. II. August 1968 , during recovery. No repetitive complexes. Irregular background activity, slow for his age. Slow wave focus with some sharp waves in the right frontal, central, and temporal region. III. April 1970, during continued remission. Much 8 to $10 \mathrm{~Hz}$ alpha activity. Slow wave focus with some sharp waves in the right parietal and occipital regions. No repetitive complexes.

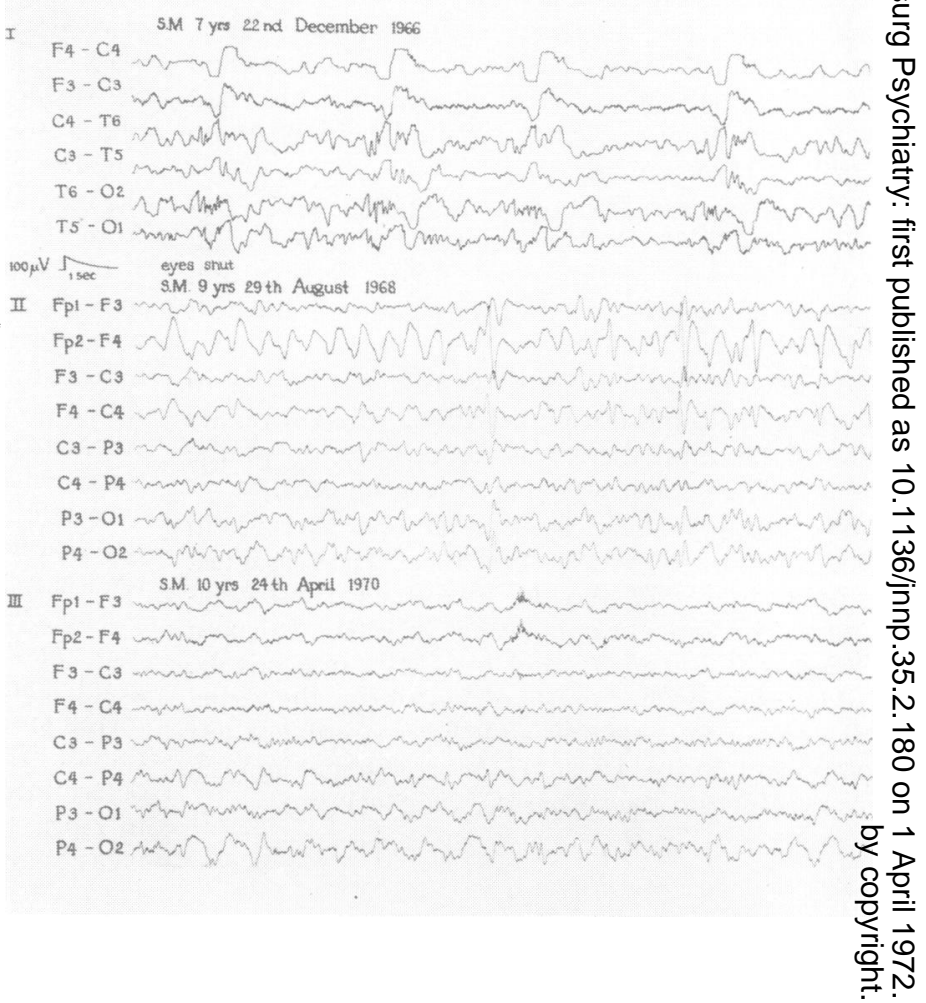

The severity of the disease does not seem to have predictive value regarding recovery or long-lasting remission. Case 2 had the disease in a severe form progressing to stage IIB of Freeman (1969), while case 1 had only slight neurological symptoms besides the convulsions and falling fits. Of the three patients described in more detail in the literature, one was a severe case (Resnick et al., 1968) and two had only slight neurological symptoms (Cobb and Morgan-Hughes, 1968).

It seems possible to diagnose the disease also in the quiescent or recovery stage. The titres of measles antibody and the platelet agglutination titre, which possibly is characteristic of slow virus infections (Palosuo and Pettay, 1970), were still high in case 2 two and a half years after recovery began, and the amount of gamma globulin was still high in the cerebrospinal fluid. Several tests may be necessary, however, as the results of the laboratory tests may vary from time to time (Kolar, 1968). On the other hand, EEG findings are not reliable. The periodic complexes may have disappeared or they are difficult to recognize as they may occur irregu- larly and their shape is not so distinct as in the characteristic EEG of the earlier stages. Reappraisal of EEGs recorded during those stages of the disease may be rewarding, however. Even $\frac{\circ}{\Phi}$ during the relapse of the disease in case 1 the EEG was not characteristic. We have no experience of the possible persistence of the $\gamma-\mathrm{C}$ fraction of the globulins in the quiescent or recovery stage. This fraction has been demonstrated by Bergmann, Dencker, Johansson, and Svennerholm (1968) in the cerebrospinal fluid 0 and by Jabbour, Garcia, Lemmi, Ragland, Duenas, and Sever (1969) in the serum of SSPE patients.

Comprehensive psychological tests may be은 helpful in diagnosing SSPE both in the active $\frac{7}{0}$ and in the recovery stage. The findings in the two cases of Cobb and Morgan-Hughes (1968) N and in our two cases show a marked difference $N$ between the results of verbal and performance $\overbrace{\mathrm{W}}$ tests. The results of the verbal tests were rela- 0 tively good, while the results of the performance tests were poor. This discrepancy appears to be most pronounced in the active stage of the $\stackrel{\infty}{+}$ disease. 
The high measles antibody titre and the high level of gamma globulins in the cerebrospinal fluid in case 2 and the progressive mental deterioration in case 1 seem to show that the disease is not overcome in the stage of recovery but is still latent. The relapse in case 1 supports this assumption.

\section{REFERENCES}

Bergmann, L., Dencker, S. J., Johansson, B. C., and Svennerholm, L. (1968). Cerebrospinal fluid $\gamma$-globulins in subacute sclerosing leucoencephalitis. Journal of Neurochemistry, 15, 781-785.

Cobb, W. A., and Morgan-Hughes, J. A. (1968). Non-fatal subacute sclerosing leucoencephalitis. Journal of Neurology, Neurosurgery, and Psychiatry, 31, 115-123.

Freeman, J. M. (1969). The clinical spectrum and early diagnosis of Dawson's encephalitis. Journal of Pediatrics, 75, 590-603.

Jabbour, J. T., Garcia, J. H., Lemmi, H., Ragland, J., Duenas, D. A., and Sever, J. L. (1969). Subacute sclerosing panencephalitis. A multidisciplinary study of eight cases. Journal of the American Medical Association, 207, 22482254.

Kennedy, C. (1968). A ten-year experience with subacute sclerosing panencephalitis. Neurology (Minneap.), 18, part 2, 58-59.

Kolar, O. (1968). Immunopathologic observations in subacute sclerosing panencephalitis. Neurology (Minneap.), 18, part 2, 107-111.

Kurtzke, J. F. (1956). Inclusion body encephalitis. A nonfatal case. Neurology (Minneap.), 6, 371-376.
Landau, W. M., and Luse, S. A. (1958). Relapsing inclusion encephalitis (Dawson type) of eight years' duration. Neurology (Minneap.), 8, 669-676.

Legg, N. J. (1967). Virus antibodies in subacute sclerosing panencephalitis: a study of 22 patients. British Medical Journal, 3, 350-352.

Pampliglione, G. (1964). Polymyographic studies of some involuntary movements in subacute sclerosing panencephalitis. Archives of Disease in Childhood, 39, 558-563.

Palosuo, T., and Pettay, O. (1970). Platelet aggregation in various types of measles virus infections (abstract.) Scandinavian Journal of Clinical and Laboratory Investigation, 25, Suppl. 113, 105.

Pearce, J. M. S. and Barwick, D. D. (1964). Recovery from presumed subacute inclusion-body encephalitis. British Medical Journal, 2, 611-613.

Penttinen, K., and Myllylä, G. (1968). Interaction in human blood platelets, viruses and antibodies. I. Platelet aggregation test with microequipment. Annales Medicinae Experimentalis et Biologiae Fenniae, 46, 188-192.

Pettay, O., Donner, M., Halonen, H., Palosuo, T., Salmi, A., and Tiilikainen, A. (1971). Subacute sclerosing panencephalitis. Preceding intellectual deterioration and aberrant measles serology. Journal of Infectious Diseases, 127, 439.

Resnick, J. S., Engel, W. K., and Sever, J. L. (1968). Subacute sclerosing panencephalitis. Spontaneous improvement in a patient with elevated measles antibody in blood and spinal fluid. New England Journal of Medicine, 279, 126-129.

Simpson, J. A. (1961). Subacute inclusion-body encephalitis: a possible association with infective hepatitis. Lancet, 2, 685-687.

Zeman, W., and Kolar, O. (1968). Reflections on the etiology and pathogenesis of subacute sclerosing panencephalitis. Neurology (Minneap.), 18, part 2, 1-7. 